\title{
Prototipo didáctico para el aprendizaje de la mecánica en los laboratorios de física universitaria mediante un sistema de adquisición y procesamiento de datos
}

\author{
Yubiry González ${ }^{a b}$, Emery Dunia ${ }^{a} \&$ Jesús Pérez $^{a b}$ \\ a Postgrado en Instrumentación, Universidad Central de Venezuela, Caracas, Venezuela.emerydunia@gmail.com, profesorjesusperez@gmail.com \\ ${ }^{b}$ Dpto. de Ciencias Básicas, Universidad Politécnica Territorial del Estado Aragua “Federico Brito Figueroa”, Maracay, Venezuela. Yubiry.gonzalez.17@gmail.com
}

\begin{abstract}
Resumen - Se presenta el diseño y construcción de un prototipo didáctico con la realización de seis trabajos prácticos de laboratorio que abarcan los contenidos curriculares de mecánica en la física básica a nivel universitario. El prototipo propuesto emplea las modernas concepciones didácticas en la enseñanza de la ciencia e incorporan las tecnologías de adquisición y procesamiento de datos mediante la utilización de un sensor ultrasónico de posición y un microcontrolador PIC unido a una interfaz de comunicación USB. El diseño de las experiencias prácticas automatizadas con la utilización de técnicas de adquisición de señales y procesamiento de datos, mostró su eficacia y versatilidad para la ejecución de experiencias prácticas en los laboratorios de Física universitarios.
\end{abstract}

Palabras Clave - trabajo práctico de laboratorio, sistema de adquisición y procesamiento de datos, laboratorio de física.

Recibido: 12 de marzo de 2017. Revisado: 20 abril de 2017. Aceptado: 3 de mayo de 2017.

Teaching prototype for the learning of mechanics in university physics laboratories through a data acquisition and processing system

Abstract- The design and development of a didactic prototype is presented along with six lab practices that comprehend the curricular contents of mechanics in basic physics university level. The proposed prototype is built upon the modern didactic concepts for the teaching of science, it also incorporates technologies for acquisition and processing data through the use of an ultrasonic position sensor and a PIC microcontroller joint with a USB communication interface. The design of automated practical experiences using signal acquisition techniques and data processing proved its efficiency and versatility on executing practical experiences in university physics laboratories.

Keywords - practical laboratory work, data acquisition and processing system, laboratories.

\section{Introducción}

La enseñanza de la física como ciencia natural, precisa de la realización de los trabajos prácticos de laboratorio (TPL) para el aprendizaje significativo de los educando. En los planes de estudios universitarios, comunes en las primeras etapas de formación de la ingeniería, se incluyen los laboratorios de física con TPL que comprenden, entre otros, los tópicos de mecánica, hidrostática, electricidad, magnetismo, óptica y física moderna. Los TPL referidos a la mecánica, la mayoría de las veces, emplean las técnicas de muestreo, tabulación y procesamiento de datos en forma manual y la repetición continua de medidas de una misma situación física particular [1-3]. El tiempo efectivo de “experimentación” para el estudiante se circunscribe al muestreo estadístico y la lectura visual de instrumentos de medición, soslayando la interpretación, análisis y variación de otros parámetros experimentales [4].

Esta dificultad de los TPL puede ser superada al incorporar las técnicas de automatización-control y adquisición-procesamiento de datos, mediante la tecnología de Microprocesadores [2,5-7] también conocidos como controladores de interfaz periférico o por su acrónimo anglosajón PIC (Peripheral Interface Controller); no solo para hacer más eficiente el procesamiento de datos y la ejecución de los ensayos, sino también; y sobre todo; para capacitar a los futuros ingenieros en el manejo de las actuales tecnologías de información y comunicación, además de los sistemas automatizados basados en PIC [8].

En este sentido, la incorporación de la tecnología de microcontroladores en los laboratorios de física serviría para implantar la pedagogía de Edgar Morín [9] según la cual, la educación es educación para la vida aquí y ahora. Siendo, el empleo de esas tecnologías en los laboratorios básicos de Física, un medio para la capacitación de los profesionales del siglo XXI. Adicionalmente, la ejercitación de los estudiantes en los TPL con microprocesadores incorpora la concepción constructivista del aprendizaje [10] pues la manera de adquirir el conocimiento es mediante la exploración y la manipulación activa de objetos e ideas, abstractas o concretas del mundo físico y social del cual somos protagonistas.

Por otro lado, cada vez es más frecuente el uso del computador en el laboratorio, bien como sistema de control y visualización de sensores físicos y de adquisición de datos, y también como herramienta para realizar simulaciones, construir gráficas que muestran la relación entre variables o realizar cálculos y ajustes de diferente tipo que ayudan al estudiante en el desarrollo de la experiencia [2,4-7]. Esta aplicación de los ordenadores puede ser muy útil en la enseñanza experimental de la física, de la química o de la tecnología, a nivel básico y avanzado, ya que puede servir de

Como citar este artículo: González, Y., Dunia, E. and Pérez, J., Prototipo didáctico para el aprendizaje de la mecánica en los laboratorios de física universitaria mediante un sistema de adquisición y procesamiento de datos. Educación en Ingeniería, 12(24), pp. 9-14, Julio, 2017. 
introducción al interesante dominio de la automatización que tiene tanta importancia en la vida moderna [8].

El objetivo del presente trabajo es presentar el diseño y construcción de un prototipo didáctico con tecnología PIC, a ser implementados en los laboratorios de física general, específicamente en los tópicos referidos a la mecánica. El prototipo consta de: 1) Una maqueta o estructura de soporte para experimentación, 2) sistema sensor de posición ultrasónico y 3) control electrónico con microcontrolador PIC.

Los detalles de su construcción se muestran en la sección de metodología. El diseño de los trabajos prácticos se especifica en la sección de resultados. La evaluación del prototipo se discute en la última sección.

\section{Metodología}

El sistema de medición consiste en un Prototipo Experimental de adquisición de datos (Hardware) y un software en Visual Basic para el procesamiento de la información. La maqueta de experimentación pone a prueba la situación física, el sensor registra la información de posición y la envía al PIC: el microprocesador crea un archivo .tex y la envía al ordenador a través del puerto USB; el ordenador crea una base de datos por medio de un software en visual basic Microsoft ${ }^{\circledR}$ que puede ser leído y procesado por cualquier programa de graficación y cálculo, como Excel $\AA^{\circledR}$, Origin ${ }^{\circledR}$; Matlab ${ }^{\circledR}$, MAPLE $®$ (Fig. 1).

Para el diseño del prototipo se emplearon los criterios de:

i) versatilidad: Empleo del mismo prototipo para la ejecución de todas las prácticas. Se ideó la maqueta y el sistema sensor para ser usados en todas y cada una de las prácticas propuestas sin modificación.

ii) Robustez: Diseño compacto que permite la manipulación por los no expertos. Se presenta el prototipo armado y su configuración permite adicionarle accesorios de forma modular de acuerdo a la aplicación. Evitando el uso de calibración previa por parte de los usuarios.

iii) Universalidad: Puede acoplarse a cualquier PC, Laptop o Tablet y permite el procesamiento de la información emanada por el sensor usando cualquier software de graficación y manejo de base de datos.

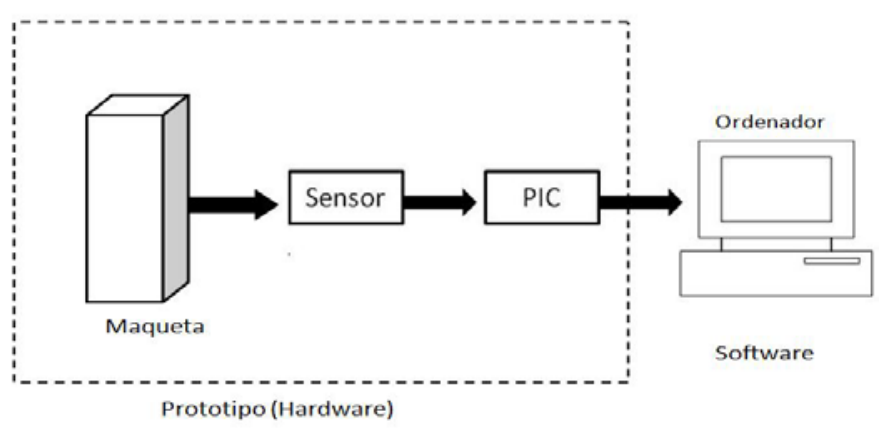

Figura 1. Configuración del sistema de Medición. El Prototipo incluye la maqueta de experimentación, el sensor de posición ultrasónico y el sistema de control electrónico, el Software consiste en un programa en visual Basic y los programas de graficación y cálculo.

Fuente: Los autores iv) Economía: El prototipo no requiere insumos adicionales, tampoco de refacciones; se ha evitado el empleo de piezas y partes móviles que se desgasten o deterioren por su manipulación. Todo el Sistema de adquisición de datos es energizado por el ordenador, no requiriendo fuentes de voltaje adicionales.

A continuación se describen los tres elementos que conforman al prototipo: 1) maqueta soporte para experimentación, 2) sistema Sensor y 3) control electrónico.

1) Maqueta: Consiste en un soporte elaborado en policloruro de vinilo de $3 \mathrm{~mm}$ de espesor en la configuración y dimensiones que se muestran en la Fig. 2. Todas las piezas y partes están unidas mediante flejes metálicos, tuercas y tornillos, evitando el uso de pegamentos, pinturas o emulsiones que se deterioren con el tiempo. El conjunto de accesorios para los distintos TPL de la maqueta de experimentación se especifican en la Fig. 2, En la sección siguiente (Resultados) se muestran los detalles de uso y especificidades.

2) Sistema sensor: Consta de un sensor de ultrasonidos HCSR04, que opera a la frecuencia de $40 \mathrm{KHz}$ para estimar distancias por medio de la medición de ecos. La principal ventaja de estos tipos de sensores es que no necesitan contacto físico para poder detectar las posiciones, con una resolución de $0,3 \mathrm{~cm}$, hasta 5 metros de alcance. El sensor tiene un retardo en el orden de 3,5 milisegundos, entre la recepción y emisión del eco, al recorrer la distancia máxima del riel de la maqueta $(0,6 \mathrm{~m})$, pero, este lapso es despreciablemente pequeño en comparación con los tiempos característicos de las situaciones experimentales propuestas (sección de resultados) del orden de los segundos o mayores.

3) Control Electrónico: Consiste en un Microcontrolador PIC 16F870 temporalizado con un oscilador de $20 \mathrm{MHz}$, para registrar las eco-posiciones del sistema sensor y una interfaz de comunicación con el ordenador; esta última es un circuito estándar empleando el integrado FT232RL (Fig.3). Adviértase que el Microprocesador así configurado, admite varias otras entradas de sensores, analógicas y digitales, para ulteriores aplicaciones.

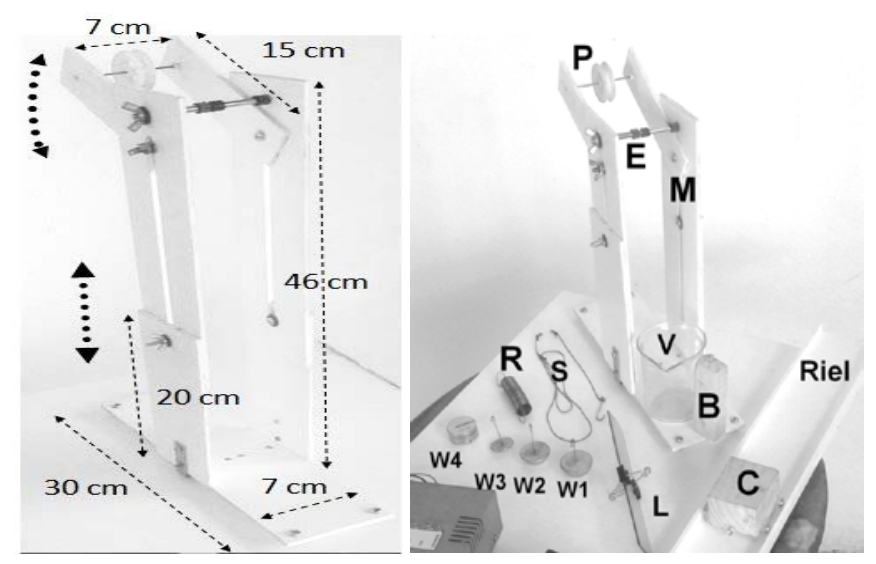

Figura 2. Maqueta de Experimentación: Dimensiones (izq) y Accesorios (der) Polea P, eje E, soporte M, resorte R, Riel de $60 \mathrm{~cm}$, móvil C, Pesos W1, W2, W3 y W4; cordel S, Lamina L; Vaso V, bloque de madera B.

Fuente: Los autores 


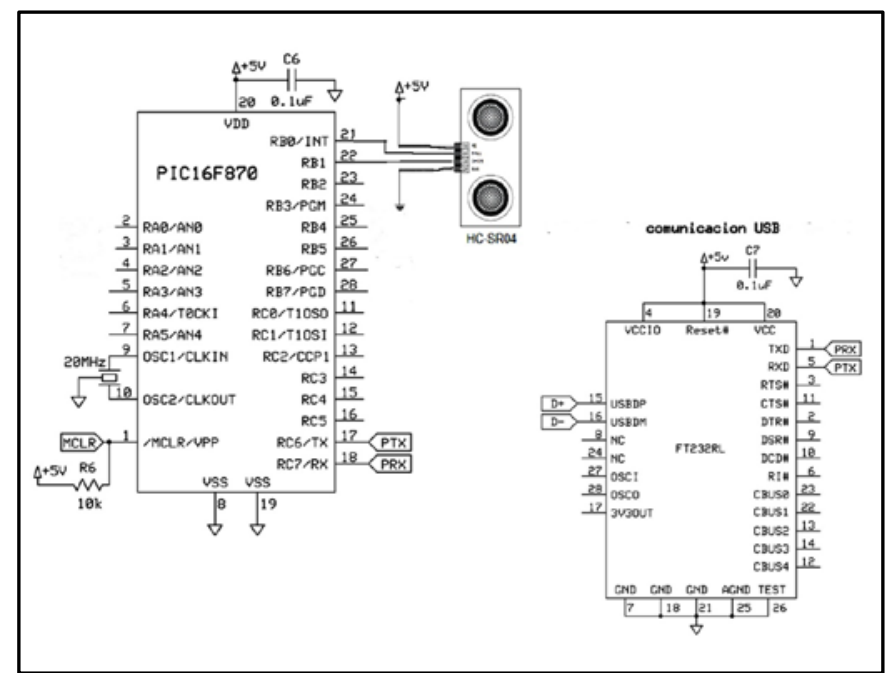

Figura 3. Control electrónico: Conexiones del PIC 16F870 (izq.) y la interface de comunicación vía puerto USB FT232RL (der), las salidas D+ y D- permiten la comunicación con el puerto USB del ordenador.

Fuente: Los autores

El software codificado en Visual Basic es una interfaz de usuario On-Off para la comunicación entre el PIC y el ordenador, permite especificar los intervalos de muestreo del sensor de posición, desde 1, 5, 10, 100, 500 y $1000 \mathrm{~ms}$. Generando en cada caso una base de datos de 2 columnas de registros: posición-tiempo en un archivo de texto (dat). Posteriormente el usuario puede abrir los registros en el ordenador con cualquier programa de graficación y cálculo para efectuar en cada caso las operaciones que modelan la situación física particular.

\section{Resultados}

El prototipo se puede emplear en la realización de varias experiencias de trabajos prácticos de laboratorio de física a nivel universitario. Como ejemplo se muestran a continuación su uso para abarcar varios contenidos de mecánica.

\subsection{Cinemática (Plano inclinado)}

Este TPL, frecuente en casi cualquier laboratorio de física general, consiste en medir la aceleración de un cuerpo que cae sobre un plano inclinado, bajo la acción de la gravedad siguiendo las ideas de Galileo para ilustrar la caída libre. Básicamente, se obtiene la gráfica de posición versus tiempo variando el ángulo de inclinación del plano y la masa del móvil. En las prácticas tradicionales no automatizadas, se obtienen de forma discreta estas gráficas, repitiendo innumerables veces las medidas. Además de monótono, la práctica suele resultar larga en duración y con muchos errores de apreciación. En este caso, se propone efectuar de forma automatizada y continua la gráfica de posición-tiempo. La mayor actividad de los estudiantes, será discutir y analizar las diferentes gráficas obtenidas, según las variables involucradas (masa, ángulo, altura) y no como en las prácticas tradicionales en la repetición de la misma situación física.

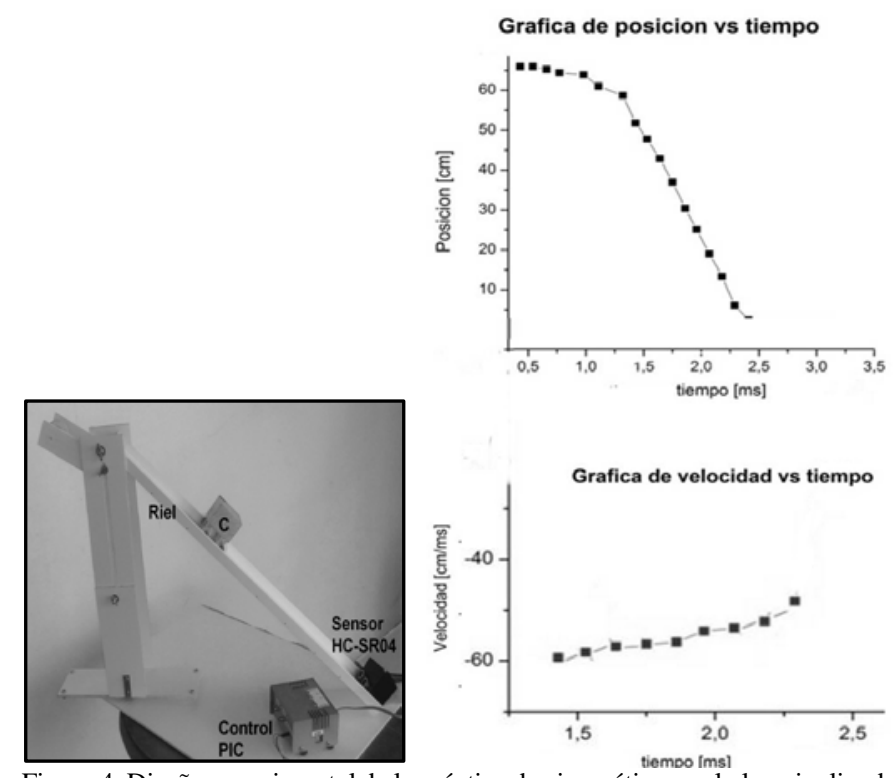

Figura 4. Diseño experimental de la práctica de cinemática en el plano inclinado (Izq). El sensor s registra la posición $\mathrm{x}$ del móvil $\mathrm{C}$ en cada instante $\mathrm{t}$ y el software en el ordenador genera las gráficas de variación de la posición y velocidad del móvil (der.)

Fuente: Los autores

\subsection{Dinámica (Ley de Hooke)}

El prototipo puede emplearse también en la ejecución del TPL sobre la Ley de Hooke, en este caso el sensor ultrasónico determina la posición final del sistema $(\mathrm{W}+\mathrm{L})$. El sensor registra la posición de equilibrio para cada masa. El estudiante varia la masa $\mathrm{W}$ para un mismo resorte $\mathrm{S}$ de constate de elasticidad $\mathrm{k}$, desconocida. Y a partir de la gráfica de elongación ( $\left.\mathrm{y}-\mathrm{y}_{0}\right)$ versus masa $(\mathrm{W}+\mathrm{L})$ se determina la constante $\mathrm{k}$. Los estudiantes pueden cambiar el resorte y obtener varios ejemplos para su discusión y comparación. La función de la lámina acrílica (L) es reflejar el eco del sensor y determinar la elongación del resorte para cada pesa.

La experiencia permite la evaluación de la constante de elasticidad del resorte, empleado en el prototipo, que resulta del orden de $\mathrm{k}=148.860[\mathrm{~N} / \mathrm{m}] \pm 10$. El coeficiente de correlación de Pearson muestra la bondad del ajuste lineal y la precisión obtenida. La posición negativa dada por el sensor, debe interpretarse como consecuencia de haberse usado la referencia de alturas en el pivote o eje del resorte, de forma que el sensor
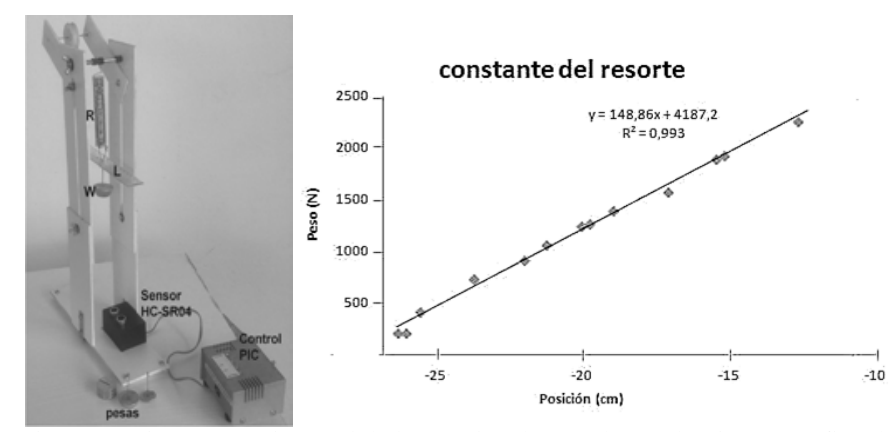

Figura 5. Diseño experimental de la práctica de Ley de Hooke (izq.), Gráfica de la constante del resorte (der).

Fuente: Los autores 
HC-SR04 mide la posición relativa desde la base de la maqueta. Debe advertirse que la lámina acrílica $L$, de masa 21,65 g \pm 0,01 g tiene por función servir de superficie reflectante para los eco de la señal generada por el sensor y se mueve conjuntamente al último alabe del resorte $\mathrm{R}$, del cual está suspendida rígidamente. Las masas empleadas como pesas en la experiencia son combinaciones de masas del orden de 110, 70, 30 y 20 gramos respectivamente.

\subsection{Dinámica (Leyes de Newton)}

Además de la verificación experimenta de la "ley" de Hooke, discutida en el apartado anterior, suele emplearse también en las prácticas de Laboratorio de Física Básica a nivel medio y Universitario, las prácticas que emplean diversas masas unidas a poleas y/o desplazándose sobre un plano inclinado. Una posible configuración para la realización de un Trabajo Práctico de Laboratorio es la mostrada en la Fig. 6.

Para cada pesa "W" el sensor registrará la variación temporal de $\mathrm{x}$, es decir, se obtendrá la gráfica de la posición instantánea del móvil C. Considerando la polea ideal y las cuerdas inextensibles, la aceleración del móvil será la aceleración del sistema, establecido por la siguiente expresión:

$$
\boldsymbol{a}=\boldsymbol{g}\left[\frac{M(\sin \varphi-\mu \cos \varphi)-m}{m+M}\right]
$$

Donde m es la masa de las pesas y M la masa del móvil C. Es importante notar que el desplazamiento máximo del móvil C es del orden de $60 \mathrm{~cm}$ (longitud del riel) y que el ángulo puede variarse a conveniencia elevando la altura del soporte $\mathrm{M}$. Adicionalmente puede variarse el coeficiente de rozamiento $(\mu)$ utilizando distintos materiales sobre la superficie del plano inclinado, como papel de lija, papel de aluminio, entre otras. El estudiante obtendrá en el ordenador las gráficas de posición versus tiempo, velocidad versus tiempo y aceleración versus tiempo, en función de las distintas variables involucradas $(\mathrm{M}, \varphi, \mu)$.
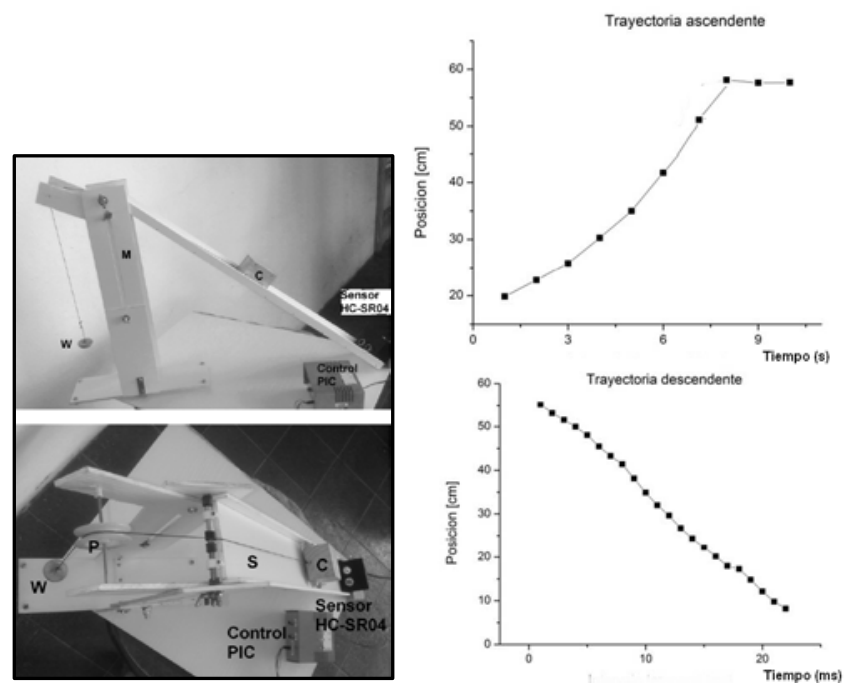

Figura 6. Diseño experimental de la práctica de Dinámica (Leyes de Newton). Las gráficas de posición generadas por el ordenador se aprecian en la figura derecha para ambas trayectorias de ascenso y descenso dependiendo del equilibrio entre la masa W y el móvil C.

Fuente: Los autores

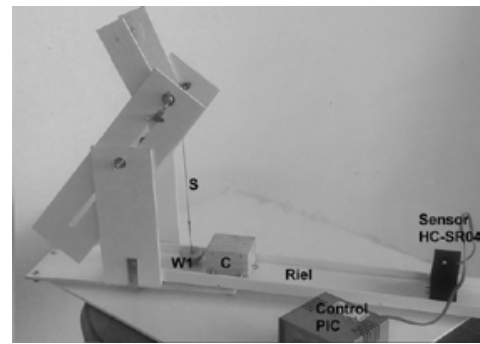

Figura 7. Diseño experimental de la práctica de Conservación de la energía y del momentum.

Fuente: Los autores

\subsection{Conservación de la energía y momentum}

En la Fig. 7, se muestra otra configuración del prototipo, para determinar la posición instantánea y por ende, la velocidad de un móvil C (de masa M) que desplaza sobre una superficie horizontal después que la masa W1 (lenteja del péndulo inicialmente en posición $\varphi$ ) le transfiere una cierta cantidad de movimiento en una colisión elástica (ideal).

El péndulo W1 (de masa m), partiendo inicialmente desde el ángulo $\varphi$ impacta a la masa C que recorrerá la distancia $x_{0}$. El sensor $s$ registrará la posición del móvil C (de masa M) en todo instante. Luego se puede evaluar la velocidad inicial del móvil C; despreciable el roce del móvil C con la superficie; y comparar con los datos experimentales.

$$
v_{C}=\frac{m}{M} \sqrt{2 g l(1-\cos \varphi)}
$$

\subsection{Empuje hidrostático}

La configuración experimental usada para la experiencia de Dinámica (Ley de Hooke), puede emplearse para estudiar el principio de Arquímedes y la noción de peso "aparente”. Así, sumergiendo el bloque $\mathrm{B}$, masa $\mathrm{m}$, en un fluido de densidad $\mathrm{r}$, como indica la Fig. 8; puede determinarse el empuje hidrostático. Si se conoce la constante $\mathrm{k}$ del resorte, el volumen de la masa $\mathrm{m}$ y el desplazamiento de la masa $\mathrm{M}$, es posible determinar experimentalmente la densidad del fluido mediante la relación:

$$
\rho=\frac{\mathrm{k}}{\mathrm{gV}_{\mathrm{m}}} \Delta \mathrm{y}
$$

Donde $\Delta y$ es la diferencia de posición detectada por el sensor ultrasónico y $\mathrm{V}_{\mathrm{m}}$ es el volumen de la masa sumergida. El estudiante verificará la validez del principio de Arquímedes y probará la dependencia del empuje hidrostático para distintos fluidos, tales como, aceite, agua, glicerina, etc.

Para el bloque de madera $\mathrm{B}$, de masa $(29,21 \mathrm{~g} \pm 0,01)$ sumergido en agua, la compresión del resorte debida al empuje sobre el sistema bloque B y lamina L (Fig. 8), resultó en $-1,68 \mathrm{~cm} \pm 0,01$; empleando el mismo resorte de la experiencia 2, cuidando que el nivel del líquido estará siempre por debajo de la posición del resorte de la masa M, dentro del envase V. Luego el empuje hidrostático para fluidos de densidad del orden o mayor que el agua, y usando masas y resortes como los empleados, resulta lo suficiente como para producir compresiones en el orden de los centímetros y por ende 


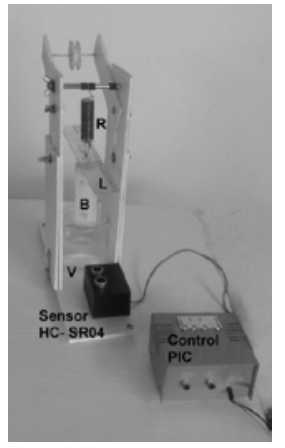

Figura 8. Diseño experimental de la práctica de empuje hidrostático. Detalles en el texto.

Fuente: Los autores

pueden ser medidas por el prototipo presentado; resultando útil como experiencia de aplicación del empuje sobre cuerpos sumergidos

\subsection{Oscilaciones amortiguadas}

Para estudiar las oscilaciones amortiguadas en un sistema masaresorte vertical, sometido a la aceleración de gravedad g, basta con registrar la posición instantánea de la masa $\mathrm{M}+\mathrm{m}$, de acuerdo a la configuración de la Fig. 5. El sensor HC-SR04 registra la posición durante la oscilación y permite además elaborar las gráficas instantáneas de velocidad y aceleración. El resorte, de constante $\mathrm{k}$ se elonga desde una longitud inicial yo hasta la posición máxima ymax. El sensor s registrará la posición en función del tiempo, y la velocidad en función del tiempo de la oscilación amortiguada:

$$
y=y_{\max } e^{-\frac{b}{2(M+m)} t} \cos (\omega t)+\frac{g}{k}(M+m)
$$

Donde b es la constante de amortiguamiento y la frecuencia de oscilación está dada por:

$$
\omega=\sqrt{\left(\frac{b}{2 M+2 m}\right)^{2}-\frac{k}{M+m}}
$$

\section{Conclusiones}

El prototipo elaborado, mostró su eficacia como sistema automatizado para la adquisición de datos de posiciones, velocidades y aceleraciones en los trabajos prácticos de laboratorio de física para la enseñanza universitaria de los contenidos de mecánica, como se mostró en la sección de resultados.

En particular su empleo para las determinaciones de las variaciones temporales de posición, mediante el uso del sensor HCSR04, mostró ser suficientemente sensible incluso para variaciones tan pequeñas como 0,2 cm en rango de distancias de 1 a $60 \mathrm{~cm}$ de longitud; como se evidenció en las experiencias de cinemática (3.1), Ley de Hooke (3.2) y Empuje Hidrostático (3.5). Se destacan las prestaciones del sensor en cuanto a su empleo en cualquier condición de iluminación y para móviles en trayectorias cortas, del orden de los centímetros, que difícilmente pueden ser alcanzadas con similar prestación por los sensores optoelectrónicos, basados en interruptores de luz infrarrojos.
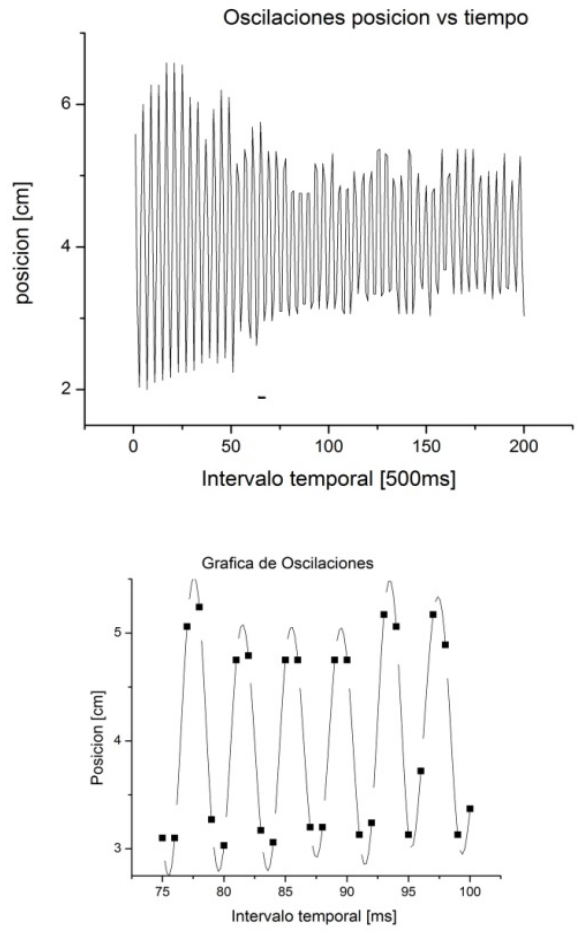

Figura 9. Gráficas del muestreo de 400 datos para el movimiento oscilatorio amortiguado en el lapso de tan solo 2 minutos. El análisis de los datos en el ordenador permite graficar las oscilaciones en periodos del orden de hasta 5 milisegundos.

Fuente: Los autores

Vale destacar que el retardo ocasionado por el tiempo de emisión-recepción del haz ultrasónico, del orden de los 3.5 milisegundos, es despreciablemente pequeño para las aplicaciones didácticas, donde los tiempos característicos de los movimientos son del orden de los segundos. Incluso para movimientos más rápidos, como es el caso del TPL sobre la dinámica de una máquina de Atwood (3.3) donde los lapsos característicos son tan breves como 0,35 segundos, el dispositivo permitió la colección de más de veinte posiciones diferentes (Fig. 6), para el cálculo de la velocidad instantánea y la aceleración media.

Por otro lado el diseño de la maqueta del prototipo, evidenció su versatilidad, robustez y economía al emplearse indistintamente como soporte portátil para la ejecución de los TPL planteados.

Destaca que el empleo y uso del microcontrolador PIC y el diseño del circuito propuesto (Fig. 2) mostró varias ventajas en su implementación: i) permitió la rápida comunicación con la interfaz de usuario al emplear como canal de comunicación el puerto USB en lugar de los puertos serial-paralelo con el ordenador, ii) su versatilidad puesto que los registros de salida del PIC son tipo texto; pudiendo ser leídos y procesados por casi cualquier software de graficación, sin requerir la programación interna del PIC para efectuar los cálculos de cada TPL particular. Muchas veces se usa la memoria interna del PIC para efectuar los cálculos y procesamiento de la información, pero ello trae como desventaja que hay que recodificar internamente el PIC para casi cualquier nuevo cálculo o información y se satura además la memoria interna del microcontrolador. 
La versatilidad y uso del prototipo para la adquisición y procesamiento de gran cantidad de información en tiempos breves, quedó de manifiesto en el TPL sobre oscilaciones amortiguadas, como se observa en la Fig. 9; colectando más de cuatrocientas lecturas en solo 2 minutos para el análisis del movimiento oscilatorio.

Por último la automatización en la adquisición de datos en los TPL de Física sirve de vínculo y ejercitación al estudiante con las nuevas tecnologías de procesamiento de información y comunicación, cada vez más extendida en las áreas de las ciencias naturales y la ingeniería.

\section{Referencias}

[1] Cruz-Ardila, J., Importancia de usar tecnología en el desarrollo de prácticas de laboratorio de física mecánica. Revista Educación en Ingeniería. 6(11), pp. 1-11, 2011.

[2] Pinto, S.S., Silva, L.S.V., Tenório-de Carvalho, C.A. y Egoavil, C.J., Pêndulo simples utilizando tecnologia embarcada de baixo custo aplicado ao Ensino da Física. Lat. Am. J. Sci. Educ. 22062, pp. 2-6, 2015.

[3] Castiblanco, O. y Vizcaíno, D., La experiencia del laboratorio en la enseñanza de la física. Revista Educación en Ingeniería. 3(5), pp. 68-74, 2008.

[4] Cruz, J. y Espinoza, V., Reflexiones sobre la didáctica en física desde los laboratorios y el uso de las TIC. Revista Virtual Universidad Católica del Norte. 35, pp. 105-127, 2012.

[5] Bohorque, Z.L., Martínez, S. y Gallegos, H.A., Diseño y construcción de un prototipo autónomo para la práctica experimental de laboratorios de física. Scientia et Technica. XVII (5), pp. 155-164, 2012.

[6] Núñez-Pérez, B.E., González, J., Jaramillo, J. y Viloria, P., Cronómetro digital utilizando un microcontrolador de la familia PIC16F87X para instrumentar electrónicamente los ensayos y experimentos en laboratorios de física mecánica. Revista Colombiana de Física, 43(1), pp.105, 2011.

[7] Bermúdez, H.H., Gallego, H.A. y Bermúdez, H.F., Prototipo mecatrónico para la enseñanza y el aprendizaje del movimiento armónico simple. Scientia et technica. 3(49), pp. 245-252, 2011.

[8] UNESCO. Declaración mundial sobre la educación superior en el siglo XXI: Visión y acción. [En línea]. [Consulta: Diciembre 15 de 2016] Disponible en: http://www.unesco.org/education/educprog/wche/declaration_spa.htm

[9] Morin, E., Los siete saberes para la educación del futuro. UNESCO, 1999, pp.17-42.

[10] Ausubel, D., Psicología Educativa. Trillas S.A., 1983, 16 P.
Y. González, recibió el título de Lic. en Física en el 2012, en la Universidad de Carabobo, Valencia, Venezuela. En el 2013 ejerce como profesora en el Instituto de Tecnología de Valencia, las cátedras de Matemática I y Proyecto socio-productivo. En el 2014 se traslada a la Universidad Politécnica Territorial del Estado Aragua, adscrita al Departamento de Ciencias Básicas, donde imparte actualmente la cátedra de física. Investigadora A1 adscrita al Programa de Estimulo del Investigador del Ministerio de Educación Universitaria Ciencia y Tecnología; investigadora asociado al Laboratorio de Física de la Atmosfera de la Universidad de Carabobo y a la Maestría en Instrumentación de la Universidad Central de Venezuela, donde cursa la Maestría. Ha presentado diversos trabajos de Investigación en Congresos Especializados en el área de Instrumentación Electrónica, Acústica Musical, Física Atmosférica y Didáctica de la Física.

ORCID: 0000-0002-8993-5499

J.A. Pérez-Rodríguez, recibió el título de Ing. Electricista en 1988, en la Universidad de Carabobo, Venezuela, MSc. en Instrumentación 2001 y de Dr. en Ciencias mención Instrumentación en 2002, en la Universidad Central de Venezuela. De 1987 al 1998 trabajó como Jefe de Proyectos de Ingeniería en la Empresa Oficina Técnica de Ingeniería JRG. En ese mismo año 1987 se Inicia como profesor e investigador en la Universidad Politécnica Territorial del Estado Aragua Federico Brito Figueroa, Venezuela, hasta el presente, donde ocupa los cargos de jefe del Departamento de Extensión Universitaria, jefe del Departamento de Postgrado, subdirector administrativo de la Universidad y jefe de la División de Investigación Extensión y Postgrado, hasta el 2012 cuando se jubila y aún se mantiene como profesor e investigador. También se desempeña desde 2003 al presente como profesor en el postgrado en Instrumentación de la Universidad Central de Venezuela. Actualmente coordina el Programa Nacional de Formación en Ingeniería Eléctrica.

ORCID: 0000-0002-1578-2565

É.R. Dunia-Amair, recibió el título de Lic. en Física en 1969, en la Universidad Central de Venezuela (UCV), hasta 1970 trabajó en su Facultad de Ciencias; en Paris obtuvo en 1972 el DEA en Cristalografía y en junio de 1980 el Doctorado en Ciencias en la Universidad Pierre et Marie Curie, Paris VI. Vuelve de profesor-investigador a la UCV; interesado en la enseñanza-aprendizaje, es comisionado a la revisión de programas de la escuela básica y el ciclo diversificado ante el Centro Nacional para el Mejoramiento de la Enseñanza de la Ciencia. De 1986 a 1990 inicia el postgrado en Instrumentación, y en los años 1990 va a comisiones de cambio curricular para la Licenciatura en Física. En la actualidad coordina el Comité Académico del Postgrado en Instrumentación y mantiene su interés por la enseñanza de la ciencia a la que contribuyen sus estudios de Logosofía.

ORCID: 0000-0002-6643-9767 